\title{
Implicaciones de la edad y el país de pertenencia en la relación entre apoyo emocional percibido y depresión: un estudio transcultural
}

\author{
Antonio Riquelme ${ }^{*}$, Marta Martín Carbonell ${ }^{2}$, Juan-Manuel Ortigosa Quiles ${ }^{1}$, Rosa-Martha Meda Lara ${ }^{3}$, Raquel Pérez Díaz ${ }^{4}$ y \\ Ana I. López Navas \\ ${ }^{1}$ Facultad de Psicología Universidad de Murcia (España). \\ ${ }^{2}$ Universidad de La Habana (Cuba). \\ ${ }^{3}$ Universidad de Guadalajara (México). \\ ${ }^{4}$ Centro de Investigaciones sobre Longevidad, Envejecimiento y Salud (CITED) (Cuba). \\ 5 Universidad Católica San Antonio. Murcia (España).
}

\begin{abstract}
Resumen: En el presente trabajo nos proponemos profundizar en el estudio de las implicaciones que la edad y los factores de tipo cultural asociados a la nacionalidad, puedan presentar respecto a la relación entre apoyo social y depresión. Con este objetivo, se ha entrevistado a una muestra total de 1503 personas de edad adulta (jóvenes, adultos y personas de edad avanzada) pertenecientes a España, Cuba y México.

Para la evaluación de la depresión, se ha empleado el cuestionario CES-D, mientras que para la evaluación del apoyo emocional percibido y la satisfacción con el mismo, se aplicó un modelo estructurado de autoinforme elaborado por nosotros en el que se recoge la percepción de disponibilidad de apoyo emocional en diferentes fuentes (pareja, familiares, amigos, compañeros, vecinos) y la satisfacción con el mismo.

Los resultados obtenidos indican que la relación obtenida entre apoyo emocional percibido según distintas fuentes y los valores en sintomatología depresiva está sujeta a variaciones en función de las influencias culturales asociadas al país de pertenencia de los sujetos entrevistados, y a su edad, así como a la interacción entre ambas variables.

Palabras clave: Apoyo social, Envejecimiento, Depresión, Estudio trans-
\end{abstract} cultural.
Title: Implications of age and country of membership in the relationship between perceived emotional support and depression: a cross-cultural study.

Abstract: The purpose of this study has been to analyze the implications that the age and the cultural factors associated with the nationality, to submit to the relationship between social support and depression. With this goal, has interviewed a total sample of 1503 persons of adult age (young people, adults and elderly) belonging to Spain, Cuba and Mexico.

For the evaluation of the depression, has been used the questionnaire CES-D, while for the assessment of emotional support perceived and satisfaction with the same we applied a formal model of self-evaluation report prepared by us which gathers the perception of availability of emotional support from various sources (partner, family members, friends, colleagues, neighbors) and their satisfaction with the same.

The results obtained indicate that the relationship obtained between emotional support according to different sources and values in depressive symptomatology is subject to changes depending on the cultural influences associated to the country of origin of those interviewed, and his age, as well as the interaction between both variables

Key words: Social support, Aging, Depression, Cross-cultural study.

\section{Introducción}

Sin duda, el trabajo desarrollado por Durkheim sobre el suicidio constituye el antecedente más significativo de la literatura especializada sobre apoyo social, al poner de manifiesto que el suicidio era más frecuente entre personas que disponían de débiles lazos sociales, (Durkheim, 1951). No obstante, previo al trabajo de Durkheim existían contribuciones importantes como las realizadas por Thomas y Znaniecki (1920), quienes señalan los efectos nocivos de la emigración de campesinos polacos a las áreas urbanas de los Estados Unidos, o Faris y Dunham (1939), al estudiar las implicaciones de la desorganización social y el aislamiento interpersonal de las grandes ciudades en la distribución poblacional de la esquizofrenia. Sin embargbo, no será hasta mediados de la década de los años setenta, cuando los trabajos de Cassel y Cobb en forma separada (Casel, 1974, Cobb, 1976) introduzcan definitivamente en la comuniad científica el concepto de apoyo social, al mostrar los efectos benéficos que la disposición de recursos sociales depara a quienes se encuentran bajo situaciones adversas.

* Dirección para correspondencia [Correspondence address]: Antonio Riquelme Marín. Facultad de Psicología Universidad de Murcia. Campus de Espinardo. 30100 Espinaro (Murcia, España). E-mail: riquelme@um.es
A partir de los años ochenta del pasado siglo, aparecen nuevas perspectivas de estudio desde las que se van a desarrollar propuestas integradoras. Así, autores como Hobfol y Stokes (1988) o Kahn y Antonucci (1980), intentan delimitar conceptualmente el apoyo social resaltando la naturaleza de las relaciones sociales como recurso asociado a distintas formas de ayuda, como pueda ser la información, el afecto o el apoyo tangible. Durante este mismo periodo van a converger otras aportaciones importantes, como las realizadas por Cohen y Syme (1985), al sentar las bases de distintas líneas de trabajo respecto a la relación entre apoyo social y salud, o los trabajos realizados por Lin, Dean y Ensel, 1986, y Lin y Ensel, 1989, donde se establece una diferenciación entre las dimensiones objetiva y subjetiva en el estudio de los sistemas informales de apoyo.

A la vista de la profusión de trabajos realizados sobre el apoyo social, podemos señalar por lo tanto, que en las últimas décadas del pasado siglo, se experiementa un auge exacerbado, llegándose a hablar de un desproporcionado interés hacia el concepto de apoyo social (House y Kahn, 1985). A pesar de este interés creciente, la investigación sobre este tema, no ha estado exenta de dificultades, entre las cuales cabe destacar la falta de acuerdo en la conceptualización misma de este constructo, así como las diferencias relativas a las perspectivas de estudio, los modelos de actuación sobre la salud o los mecanismos implicados en la relación entre apoyo social y salud. 
En relación a las perspectivas de estudio en el apoyo social, es importante resaltar la coexistencia de una perspectiva estructural en la que se hace hincapié en la idea de "red social" y sus conceptos asociados (tamaño, composición, densidad, frecuencia, etc.) , una perspectiva de tipo contextual, centrada en la naturaleza de las fuentes de apoyo (pareja, familia, amigos, compañeros, vecinos, etc.), y una perspectiva de tipo funcional focalizada en el tipo de los recursos provisto por las relaciones sociales (emocional, tangible, informacional, etc.).

Por lo que se refiere a la relación entre apoyo social y salud, a lo largo de la década de los 80, se desarrollaron distintos modelos explicativos, (Lin 1986; Barrera 1986; Cohen 1988; House 1981 o Cohen y McKay, 1984). Entre todas estas aportaciones, cabría señalar como denominador común dos planteamientos fundamentales, el primero de ellos centrado en la existencia de un efecto directo del apoyo social sobre la salud y el segundo marcado por el papel amortiguador del apoyo social respecto a los efectos del estrés. Desde el primer planteamiento el nivel de apoyo social no intervendría en la relación entre estrés y salud; de hecho, estrés y apoyo social no son concebidos como elementos directamente relacionados. Desde la segunda acepción, el apoyo social no ejerce un efecto positivo en personas con bajo nivel de estrés, dado que su efecto benéfico sobre la salud, tiene que ver con la amortiguación del impacto psicológico de estresores potencialmente relevantes.

Junto a estas áreas de divergencia en la investigación sobre apoyo social, se hace necesario incluir el énfasis puesto en distintos trabajos sobre cuáles son los mecanismos de actuación a través de los cuales el apoyo social actúa sobre la salud. Diferentes factores pueden mediar en las consecuencias del apoyo social, entre los que destacan las características del propio individuo tales como la edad, el sexo o características de personalidad. Algunas de estas variables personales han recibido especial atención, como es el caso de la autoestima, el afrontamiento, el locus de control, el sentido de coherencia o las expectativas de autoeficacia. Asimismo, se hace necesario contemplar también otras variables de carácter contextual, como las propiedades específicas de las relaciones interpersonales, los factores de tipo cultural, o aspectos coyunturales como el propio estado emocional del individuo. (Aranda y Pando, 2013; Castellano, 2014; Riquelme, Martín, Carrillo y López, 2010).

Concretamente, respecto a la depresión, de forma más reciente, son muchos los trabajos que se han realizado en el intento de comprender mejor la relación entre apoyo social y depresión en muy diversas situaciones. Así, en España se ha estudiado la relación entre apoyo social y depresión en inmigrantes (Martínez, Gracia, y Maya, 2001), en adolescentes con problemas de salud (Barra, Cerna, Kramm y Véliz, 2006); en personas con enfermedad cardiovascular o en hipertensos (Calvo, Díaz, Ojeda, Ramal y Alemán, 2002). Igualmente, los estudios realizados en el ámbito internacional han contemplado un panorama especialmente amplio de situaciones. En este sentido, se ha estudiado la relación entre apoyo social y depresión en personas con estrés laboral (Chen, Siu, Lu, Cooper y Philips, 2009); estrés provocado por la amenaza terrorista (Sahar, Gohen, Grogan, Braile y Henrich 2009); la aculturación (Ayers, Hofstetter, Usita, Irvin, Kang y Hovell, 2009); la muerte de un ser querido (Burke y Neimeyer, 2010); o el trauma psicológico (Schumn, Briggs y Hobfoll, 2006). También se ha estudiado el apoyo social, relacionado con variables de personalidad como el perfeccionismo (Shery, Law, Hewitt, Flett y Besser, 2008), el optimismo (Piko, Luszczynska y Fitzpatrick, 2012), o con los más dispares problemas de salud, como infección por sida (Vyavaharkar, Moneyham, Corwin, Saunders, Annang y Tavakoli, 2010); la esclerosis múltiple (Vargas y Arnett, 2010); o procesos oncológicos (Robles, Morales, Jiménez y Morales, 2009).

Menos atención se ha prestado a las implicaciones de la edad con respecto a la relación entre apoyo social y depresión, aunque existe un amplio volumen de trabajos en los que se pone de manifiesto la influencia de la edad sobre los efectos del apoyo social. Pueden señalarse por ejemplo, los trabajos efectuados por Lynch (1995) o Nolen-Hoesksema y Aldao (2011), cuyos datos muestran cómo la capacidad del apoyo social para moderar los efectos negativos del estrés vital, es modulada tanto por factores relacionados con características de personalidad, como por la edad y el género.

Respecto a la influencia de los factores culturales sobre la disposición, valoración y funcionalidad del apoyo social, podemos decir que constituye un área de trabajo que ha recibido interés por parte de los teóricos del apoyo social de manera más reciente.

Tal vez por ello, existe una gran heterogeneidad en los planteamientos realizados por los distintos estudios o mejor dicho, en sus áreas específicas de análisis. Así por ejemplo, Glazaer (2006), en un trabajo realizado sobre más de 15000 empleados de una multinacional, analiza el papel de la cultura sobre la percepción y capacidad funcional de distintas fuentes de apoyo ante el estrés laboral, mediante la comparación de trabajadores norteamericanos (angloparlantes) europeos (occidentales y orientales), sudamericanos y asiáticos. Este trabajo puso de manifiesto la relevancia del componente individualista o colectivista de la cultura de procedencia.

Otras áreas de estudio respecto a las influencias culturales han sido la percepción del apoyo (O'Leary, Murphy y Chen, 2009), la provisión del mismo (Goodwin y Giles, 2003), o la capacidad funcional de distintas fuentes de apoyo en personas pertenecientes a distintas culturas (Brannan, Biswas-Dienerb, Mohrb, Mortazavic y Steinb, 2013).

Los trabajos anteriormente indicados, resaltan tanto la importancia del apoyo social respecto a la depresión en las más distintas situaciones, como la existencia de diferencias importantes relativas al apoyo social en función de la edad o la cultura. Sin embargo, existe una carencia importante de estudios donde se valore la interacción entre estas variables y sus implicaciones consideradas de manera conjunta, tanto respecto a los problemas de salud en general como respecto a las alteraciones depresivas en particular. 
A la vista de la revisión realizada sobre la literatura especializada en este tema, el presente trabajo pretende abordar el estudio de las implicaciones de la edad y los factores culturales relativos al país de pertenencia, en la relación entre apoyo social y depresión a partir de un estudio transcultural realizado al comparar las diferencias entre tres muestras de estudio tomadas en sus respectivos países: España, Cuba y México.

\section{Método}

\section{Participantes}

El presente trabajo está contextualizado en un estudio transcultural más amplio sobre depresión y envejecimiento a través del desarrollo adulto. De cara a los análisis realizados en el trabajo que aquí presentamos, se contó con la participación de 1503 personas pertenecientes a tres países: España, Cuba y México. Concretamente en el caso de España, participaron 503 entrevistados, $(38.90 \%$ hombres y $61.10 \%$ mujeres). En el caso de Cuba, concurrieron 330 entrevistados (39,10\% hombres y $61.10 \%$ mujeres). Por lo que se refiere a México, participaron 674 personas $(35.50 \%$ hombres y $64.50 \%$ mujeres). Podemos decir en este sentido, que no existen diferencias significativas en lo que se refiere a la proporción de hombres y mujeres en las tres muestras de estudio.

Respecto a la edad, en la muestra tomada de España, la media fue 43.74 ( $D T=21.06)$. En el caso de Cuba, la media de edad fue $52.97(D T=20.90)$, y en lo que se refiere a México, la edad media fue 46.06 (DT=24.10).

\section{Instrumentos}

Para este estudio, se trabajó con las variables país de pertenencia, edad, depresión y apoyo emocional. Para la evaluación de la depresión empleamos la Escala del Centro de Estudios Epidemiológicos de la Depresión (Center for Epidemiologic Studies-Depression Scale, CES-D) (Radlof, 1977)

La escala CES-D fue desarrollada para su uso en estudios epidemiológicos en población general. Se trata de un cuestionario de 20 ítems, con cuatro opciones de respuesta que indican la frecuencia y/o intensidad de la presentación de cada ítem.

Consideramos que este instrumento se adecúa especialmente a nuestro estudio, dado que fue diseñado para la valoración de sintomatología depresiva en población general cubriendo todo el rango del desarrollo adulto, y que han sido publicados trabajos sobre sus cualidades psicométricas en los distintos países que participan en esta investigación. (Campo, Diaz, Rueda, Cadena y Hernández, 2007; Martín, Riquelme, Pérez, Enríquez y Ortigosa, 2008; Zunzunegui, Delgado, Pérez, Yague, Illescas y León, 1998).

Para la evaluación del apoyo emocional percibido y la satisfacción con el mismo elaboramos un modelo estructurado de autoinforme en el que se pedía al individuo su percepción del nivel de apoyo emocional disponible según distintas fuentes: pareja, familiares, amigos, compañeros y vecinos. En las preguntas sobre el apoyo emocional disponible de cada una de las fuentes y sobre la satisfacción en general con el apoyo emocional disponible, se usó una escala tipo Likert de cinco puntos donde 0 significaba nada y 4 totalmente. (Véase anexo para una información más detallada)

Las distintas dimensiones de apoyo emocional valoradas mediante este instrumento, han mostrado una elevada correlación con las puntuaciones para esas mismas dimensiones del cuestionario NSSQ (Norbeck, Lindsey y Carrieri, 1983). Concretamente en un trabajo realizado previamente (Riquelme, 2012), se obtuvo una correlación de $.55(p<.000)$ en apoyo emocional de la pareja, de $.28(p=.032)$ en apoyo emocional de la familia, y de $.39(p<.000)$ en apoyo emocional de los amigos. En este mismo trabajo, la satisfacción con el apoyo emocional disponible mostró una correlación de .38 $(p<.000)$ con la dimensión de apoyo emocional del cuestionario Mos (Sherbourne y Stewart, 1991). Por último, este instrumento, ha mostrado un valor test-retest de .87 .

\section{Procedimiento}

Para la recogida de información, se aplicó el modelo general de entrevista estructurada efectuado a tal fin, a una muestra no probabilística, de manera heteroaplicada mediante la intervención de entrevistadores previamente adiestrados en el manejo de los instrumentos de evaluación.

Se estableció una diferenciación de tres grupos de edad: adultos jóvenes (de 18 a 25 años), adultos, (de 35 a 55 años) y personas de edad más avanzada (65 años o más años). Concretamente, en España participaron 201 sujetos en el primer grupo, 87 en el segundo y 261 en el tercero. Respecto a Cuba, participaron 158 entrevistados en el primer grupo, 67 en el segundo y 162 en el tercero. Finalmente, por lo que se refiere a México, se entrevistó a 144 personas para el grupo primero, 172 para el segundo y 251 para el tercero. En conjunto, la muestra la constituyeron 549 jóvenes, 387 adultos y 567 personas de edad más avanzada.

Los datos de España se tomaron de la Región de Murcia a través de heteroaplicación del modelo de entrevista tanto en población general como en asociaciones sociales, centros de día y residencias de ancianos. Por su parte, los casos de Cuba provinieron de la Ciudad de la Habana y se obtuvieron de la comunidad (municipio Cerro), centros de trabajo y estudio, asociaciones de ancianos y de los servicios del centro iberoamericano de la tercera edad. Los casos de México derivaron de Ciudad Guzmán y la Zona Metropolita de Guadalajara en Jalisco, captados en universidades, oficinas de gobierno, asilos y estancias de día.

\section{Análisis de datos}

Para estudiar las implicaciones de la edad y el país de procedencia respecto a la relación entre apoyo emocional y depresión, efectuamos distintos análisis de varianza sobre depresión atendiendo a cada una de las fuentes de apoyo 
emocional. De cara al estudio de las implicaciones en las variaciones del apoyo emocional en cada fuente, así como la satisfacción general con el mismo, hemos categorizado estas variables en tres niveles: nivel bajo que engloba a un nivel de percepción o satisfacción equivalente a nada y poco, nivel medio en el caso de quienes valoraron su nivel de apoyo/satisfacción como moderado, y nivel alto para quienes indicaron sentir mucho apoyo o sentirse totalmente apoyados emocionalmente o bien muy o totalmente satisfechos con el apoyo emocional disponible (véase Tabla 1).

Con objeto de poder precisar el sentido de las diferencias significativas obtenidas en los análisis de varianza, hemos realizado una prueba post-hoc respecto a las variables país de procedencia, edad, y apoyo social percibido, en relación a las distintas fuentes de apoyo emocional estudiadas.

\section{Resultados}

Como señalábamos anteriormente, para estudiar las implicaciones de la edad y el país de procedencia respecto a la relación entre apoyo emocional y depresión, efectuamos distintos análisis de varianza sobre depresión atendiendo a cada una de las fuentes de apoyo emocional. Puede apreciarse un resumen de los resultados obtenidos en la Tabla 1.

Tabla 1. Resumen de resultados de los análisis de varianza.

\begin{tabular}{|c|c|c|c|c|c|}
\hline Origen & Suma de cuadrados tipo III & $\mathrm{gl}$ & Media cuadrática & $F$ & $p$ \\
\hline País & 1079.815 & 2 & 539.907 & 5.857 & .003 \\
\hline Edad & 6728.782 & 2 & 3364.39 & 36.499 & .000 \\
\hline País * Edad & 10298.351 & 4 & 2574.588 & 27.930 & .000 \\
\hline Apoyo de pareja & 4539.573 & 2 & 2269.786 & 27.222 & .000 \\
\hline País * apoyo de pareja & 867.426 & 4 & 216.856 & 2.601 & .035 \\
\hline Edad $*$ apoyo de pareja & 85.700 & 4 & 21.425 & .257 & .905 \\
\hline País $*$ Edad $*$ apoyo de pareja & 1859.709 & 8 & 232.464 & 2.788 & .005 \\
\hline Apoyo de familia & 4971.107 & 2 & 2485.554 & 29.009 & .000 \\
\hline Apoyo de amigos & 5944.051 & 2 & 2972.026 & 34.704 & .000 \\
\hline País * apoyo de amigos & 799.770 & 4 & 199.942 & 2.335 & .054 \\
\hline País $*$ Edad $*$ apoyo de amigos & 1294.088 & 8 & 161.761 & 1.889 & .058 \\
\hline Apoyo de compañeros & 3868.042 & 2 & 1934.021 & 22.588 & .000 \\
\hline País * apoyo de compañeros & 1276.280 & 4 & 319.070 & 3.726 & .005 \\
\hline Apoyo de vecinos & 3782.965 & 2 & 1891.482 & 21.757 & .000 \\
\hline País * apoyo de vecinos & 1525.425 & 4 & 381.356 & 4.387 & .002 \\
\hline Satisfacción apoyo emocional & 7587.800 & 2 & 3793.900 & 45.721 & .000 \\
\hline País * satisfacción apoyo emocional & 2508.121 & 4 & 627.030 & 7.557 & .000 \\
\hline Edad * satisfacción apoyo emocional & 837.422 & 4 & 209.356 & 2.523 & .039 \\
\hline
\end{tabular}

Al considerar las variables país de pertenencia y edad, podemos señalar que las puntuaciones medias en depresión fueron superiores en España $(M=21.38, D T=7.8)$ y México $(M=22.17, D T=11.4)$ con respecto a Cuba $(M=20.04, D T=$ 11.22 ). En cuanto a la edad, la sintomatología depresiva fue más elevada en el grupo de jóvenes $(M=21.51, D T=7.68)$ y ancianos $(M=24.12, D T=11.49)$ con respecto al grupo de adultos $(M=17.53, D T=10.64)$, Como puede apreciarse en los análisis post-hoc, realizados sobre estas variables, cuyos resultados aparecen resumidos en las tablas 2 y 3, respectivamente.

Tabla 2. DSH de Tukey en depresión según el país.

\begin{tabular}{|c|c|c|c|c|c|}
\hline \multirow{2}{*}{ (I)País } & \multirow{2}{*}{ (J)País } & \multirow{2}{*}{ Diferencia de medias (I-J) } & \multirow{2}{*}{ Sig. } & \multicolumn{2}{|c|}{ Subconjunto } \\
\hline & & & & 1 & 2 \\
\hline \multirow{2}{*}{ España } & Cuba & 1.24 & .207 & \multirow[t]{2}{*}{20.04} & \\
\hline & México & -.79 & .366 & & \multirow{4}{*}{21.38} \\
\hline \multirow{2}{*}{ Cuba } & España & -1.24 & .207 & \multirow{4}{*}{21.38} & \\
\hline & México & $-2.03^{*}$ & .010 & & \\
\hline \multirow{2}{*}{ México } & España & .79 & .366 & & \\
\hline & Cuba & $2.03^{*}$ & .010 & & 21.17 \\
\hline
\end{tabular}


Tabla 3. DSH de Tukey en depresión según la edad.

\begin{tabular}{|c|c|c|c|c|c|c|c|c|}
\hline \multirow{2}{*}{\multicolumn{2}{|c|}{ (I)Edad agrupada }} & \multirow{2}{*}{\multicolumn{2}{|c|}{ (J)Edad agrupada }} & \multirow{2}{*}{ Diferencias de medias } & \multirow{2}{*}{ Sig. } & \multicolumn{3}{|c|}{ Subconjunto } \\
\hline & & & & & & 1 & 2 & 3 \\
\hline \multirow{5}{*}{ dimension2 } & 18 a 25 años & dimension3 & $\begin{array}{c}35 \text { a } 55 \text { años } \\
>64 \text { años }\end{array}$ & $\begin{array}{l}3.99 * \\
-2.61\end{array}$ & $\begin{array}{l}.000 \\
.000\end{array}$ & \multirow{2}{*}{17.53} & \multirow{5}{*}{21.51} & \\
\hline & & & 18 a 25 años & $-3.99 *$ & .000 & & & \\
\hline & 35 a 55 anos & dimensions & > 64 años & $-6.59 *$ & .000 & & & \\
\hline & \multirow{2}{*}{$>64$ años } & \multirow{2}{*}{ dimension 3} & 18 a 25 años & $2.61 *$ & .000 & & & \multirow[t]{2}{*}{24.12} \\
\hline & & & 35 a 55 años & $6.59 *$ & .000 & & & \\
\hline
\end{tabular}

Con respecto a las implicaciones recíprocas entre edad y país, en la figura 1 , se puede apreciar la existencia de una interacción significativa respecto a depresión, al verse la relación entre edad y depresión mediada por el país de pertenencia.

Respecto al apoyo emocional de la pareja (AEP, en adelante), aparecen diferencias altamente significativas $(p<.000)$. Concretamente la puntuación media en el Ces-d para quienes tienen un nivel bajo de apoyo emocional es 26.41 (DT= 11.58), mientras que en el nivel intermedio la puntuación desciende a $20.70(D T=9.07)$ y en el nivel de apoyo emocional elevado, la media en depresión es 19.06 (DT= 9.34). Como puede apreciarse en el análisis post hoc recogido en la tabla 4, los grupos de apoyo alto y medio, difieren del grupo caracterizado por un bajo apoyo emocional percibido, en el que los valores en depresión son apreciablemente más elevados.

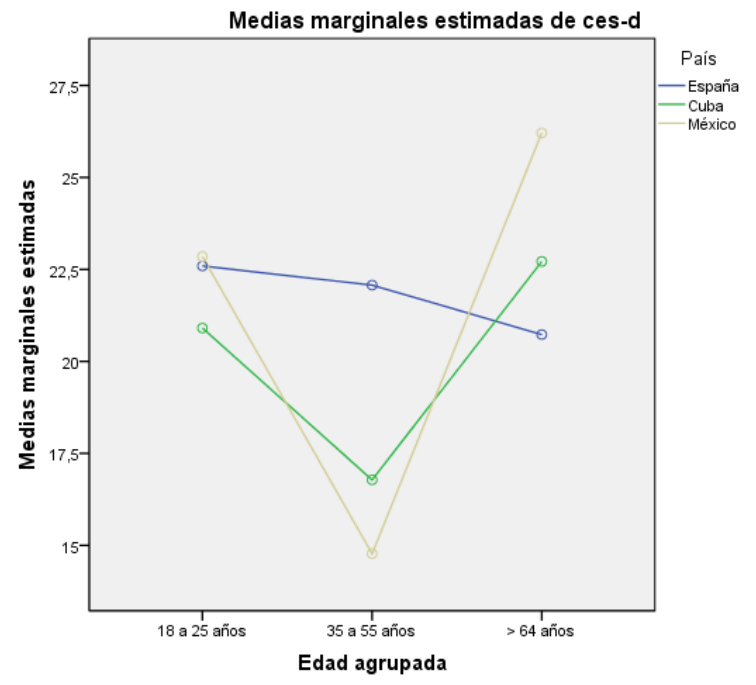

Figura 1. Interacción entre Edad y País respecto a la depresión.

Tabla 4. DSH de Tukey en depresión según apoyo social percibido. Apoyoemocional pareja $\quad$ (J)Apoyoemocional pareja

\begin{tabular}{|c|c|c|c|c|c|c|c|}
\hline \multicolumn{2}{|c|}{ 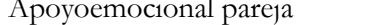 } & \multicolumn{2}{|c|}{ () Apoyoemocional pareja } & \multirow{2}{*}{$\frac{1 \text { Direrencia de medias }(1-j)}{5.71^{*}}$} & \multirow{2}{*}{$\begin{array}{l}\text { Sig. } \\
.000\end{array}$} & \multirow{2}{*}{ Apoyo Emocional } & \multirow{2}{*}{$\begin{array}{c}\text { Subconjuntos } \\
12 \\
\end{array}$} \\
\hline \multirow{6}{*}{ dimension2 } & \multirow{2}{*}{ Bajo } & \multirow{2}{*}{ dimension 3} & Medio & & & & \\
\hline & & & Alto & $7.35^{*}$ & .000 & \multirow[t]{2}{*}{ Alto } & \multirow[t]{2}{*}{19.06} \\
\hline & \multirow{2}{*}{ Medio } & \multirow{2}{*}{ dimension3 } & Bajo & $-5.71^{*}$ & .000 & & \\
\hline & & & Alto & 1.64 & .126 & \multirow[t]{2}{*}{ Medio } & \multirow[t]{2}{*}{20.70} \\
\hline & \multirow{2}{*}{ Alto } & \multirow{2}{*}{ dimension3 } & Bajo & $-7.35^{*}$ & .000 & & \\
\hline & & & Medio & -1.64 & .126 & Bajo & 26.41 \\
\hline \multicolumn{2}{|c|}{ Apoyoemocional familia } & \multicolumn{2}{|c|}{ (J)Apoyoemocional familia } & Diferencia de medias (I-J) & Sig. & Apoyo Emocional & Subconjuntos \\
\hline \multirow{6}{*}{ dimension2 } & \multirow{2}{*}{ Bajo } & \multirow{2}{*}{ dimension 3} & Medio & $3.13^{*}$ & .004 & & 123 \\
\hline & & & Alto & $7.30^{*}$ & .000 & \multirow[t]{2}{*}{ Alto } & \multirow[t]{2}{*}{19.06} \\
\hline & \multirow{2}{*}{ Medio } & \multirow{2}{*}{ dimension3 } & Bajo & $-3.13^{*}$ & .004 & & \\
\hline & & & Alto & $4.17 *$ & .000 & \multirow[t]{2}{*}{ Medio } & \multirow[t]{2}{*}{24.15} \\
\hline & \multirow{2}{*}{ Alto } & \multirow{2}{*}{ dimension 3} & Bajo & $-7.30^{*}$ & .000 & & \\
\hline & & & Medio & 4.17 & .000 & Bajo & 27.29 \\
\hline \multicolumn{2}{|c|}{ Apoyoemocional amigos } & (J)Apoyoems & igos & Diferencia de medias (I-J) & Sig. & Apoyo Emocional & Subconjuntos \\
\hline & & & Medio & $4.77^{*}$ & .000 & & 123 \\
\hline & Bajo & dimension3 & Alto & $6.76^{*}$ & .000 & Alto & 19.81 \\
\hline dimencion? & Medip & dimencion 3 & Bajo & $-4.77^{*}$ & .000 & & \\
\hline dimensionz & Medio & dimensions & Alto & 1.99 & .002 & Medio & 21.81 \\
\hline & & & Bajo & $-6.76^{*}$ & .000 & & \\
\hline & Alto & dimension3 & Medio & -1.99 & .002 & Bajo & 26.58 \\
\hline Apoyoemoci & mpañeros & (J)Apoyoems & mpañeros & Diferencia de medias (I-J) & Sig. & Apoyo Emocional & Subconjuntos \\
\hline & & & Medio & $2.94^{*}$ & .000 & & 123 \\
\hline & Bajo & dimension3 & Alto & $5.26^{*}$ & .000 & Alto & 19.06 \\
\hline dimension? & Medio & dimension 3 & Bajo & $-2.94^{*}$ & .000 & & \\
\hline dimension 2 & Medio & dimensions & Alto & $2.32 *$ & .001 & Medio & 21.38 \\
\hline & & & Bajo & $-5.26^{*}$ & .000 & & \\
\hline & Alto & dimension3 & Medio & $-2.32^{*}$ & .001 & Bajo & 24.32 \\
\hline
\end{tabular}




\begin{tabular}{|c|c|c|c|c|c|c|c|}
\hline \multicolumn{2}{|c|}{$\overline{\text { Apoyoemocional vecinos }}$} & \multicolumn{2}{|c|}{ (I)Apoyoemocional vecinos } & Diferencia de medias (I-J) & Sig. & Apoyo Emocional & Subconjuntos \\
\hline \multirow{6}{*}{ dimension2 } & \multirow{2}{*}{ Bajo } & \multirow{2}{*}{ dimension3 } & Medio & $2.37^{*}$ & .000 & & 123 \\
\hline & & & Alto & $5.27^{*}$ & .000 & Alto & 18.25 \\
\hline & \multirow{2}{*}{ Medio } & \multirow{2}{*}{ dimension3 } & Bajo & $-2.37^{*}$ & .000 & & \\
\hline & & & Alto & $2.91 *$ & .000 & Medio & 21.16 \\
\hline & \multirow{2}{*}{ Alto } & \multirow{2}{*}{ dimension3 } & Bajo & $-5.27 * *$ & .000 & & \\
\hline & & & Medio & $-2.91 *$ & .000 & Bajo & 23.53 \\
\hline \multicolumn{2}{|c|}{ Satisfacción Al emocional } & $\begin{array}{l}\text { (J)Satisfacción a } \\
\text { emocional }\end{array}$ & & Diferencia de medias (I-J) & Sig. & Apoyo Emocional & Subconjuntos \\
\hline \multirow{6}{*}{ dimension2 } & \multirow{2}{*}{ Bajo } & \multirow{2}{*}{ dimension3 } & Medio & $5.35^{*}$ & .000 & & 123 \\
\hline & & & Alto & $9.44^{*}$ & .000 & Alto & 19.53 \\
\hline & \multirow{2}{*}{ Medio } & \multirow{2}{*}{ dimension3 } & Bajo & $-5.35^{*}$ & .000 & & \\
\hline & & & Alto & $4.10^{*}$ & .000 & Medio & 23.62 \\
\hline & \multirow{2}{*}{ Alto } & \multirow{2}{*}{ dimension3 } & Bajo & $-9.44 * *$ & .000 & & \\
\hline & & & Medio & $-4.10^{*}$ & .000 & Bajo & 28.97 \\
\hline
\end{tabular}

Junto a lo anteriormente mencionado, el dato más relevante con respecto al apoyo emocional de la pareja y su relación respecto a la depresión, es la existencia de una interacción significativa con el país, así como con la edad y el país considerados en forma conjunta.

Concretamente, en el caso de la interacción entre apoyo emocional, país y depresión, encontramos mayores diferencias asociadas al alto apoyo emocional en el caso de la muestra mexicana, mientras que para esta misma muestra, el bajo nivel de apoyo emocional de la pareja, resulta menos relevante.

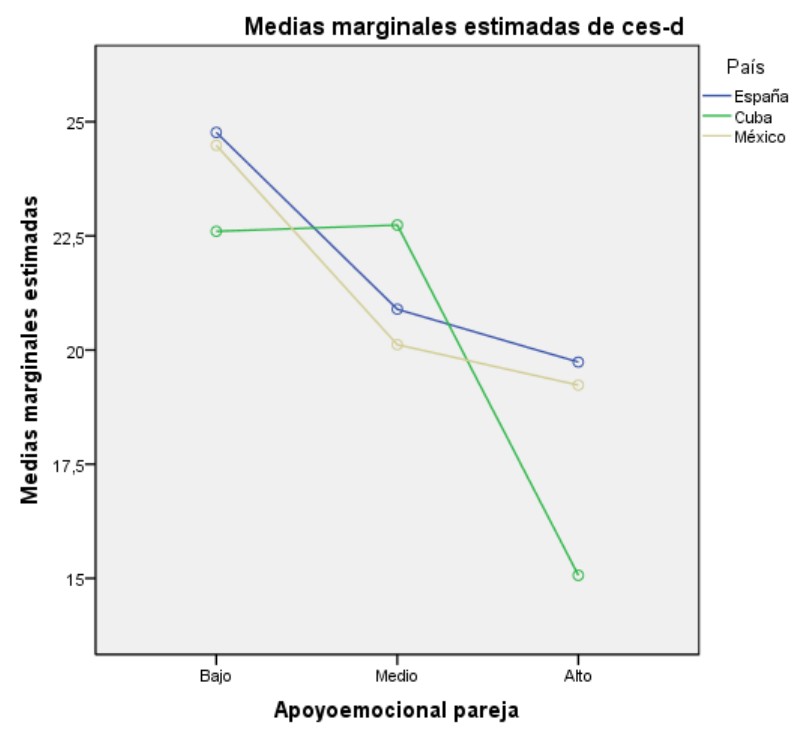

Figura 2. Interacción entre apoyo emocional de la pareja y país respecto a la depresión.

Respecto a la interacción entre AEP, país y edad sobre la depresión, puede apreciarse en la comparación de las figuras 3.1 a 3.3, cómo en unos países existen variaciones respecto a otros en cuanto a la importancia del apoyo de la pareja sobre la depresión en función del grupo de edad.

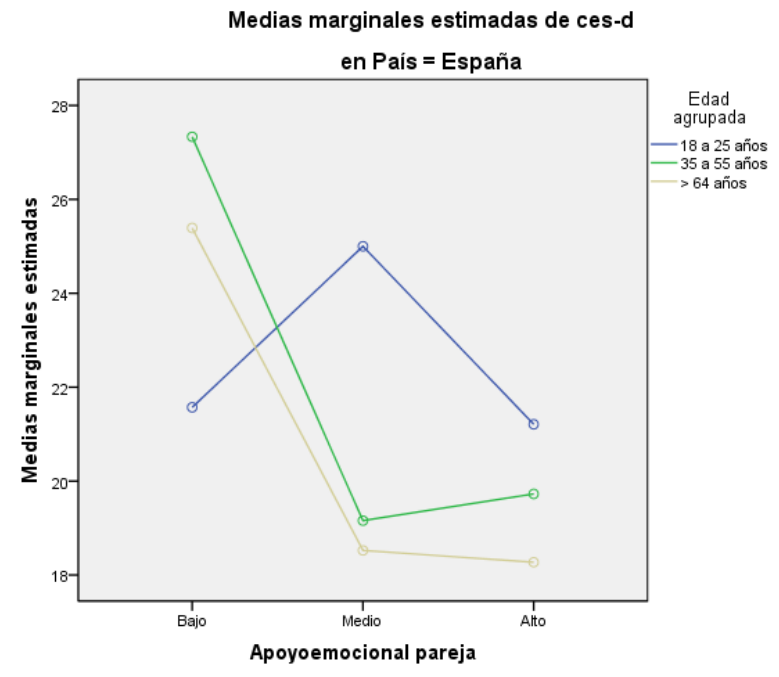

Figura 3.1. Interacción entre apoyo emocional de la pareja, edad y país respecto a la depresión (España).

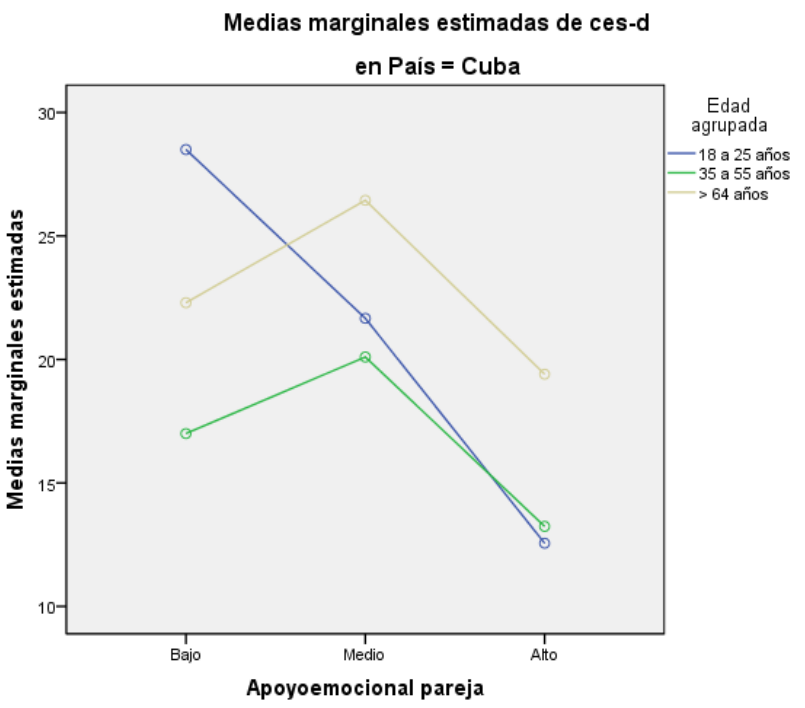

Figura 3.2. Interacción entre apoyo emocional de la pareja, edad y país respecto a la depresión (Cuba). 


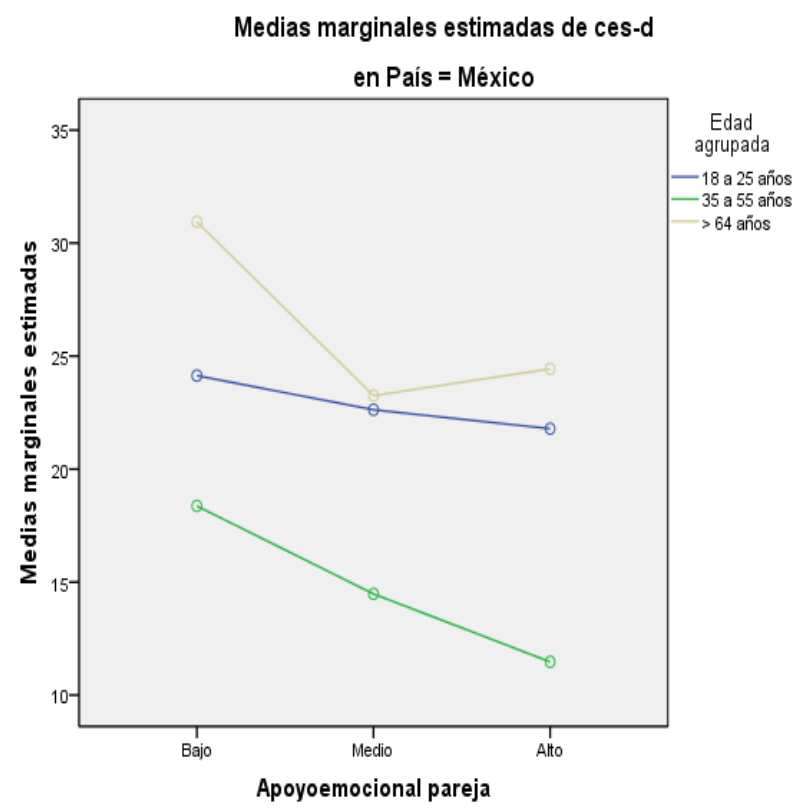

Figura 3.3. Interacción entre apoyo emocional de la pareja, edad y país respecto a la depresión (México).

En relación al apoyo emocional de la familia (AEF, en adelante), si bien se han encontrado diferencias significativas respecto a la depresión, en el sentido de que a mayor $\mathrm{AEF}$, menor depresión (véase resultados en Tabla 4), no han aparecido diferencias significativas con respecto a su interacción con el país o la edad.

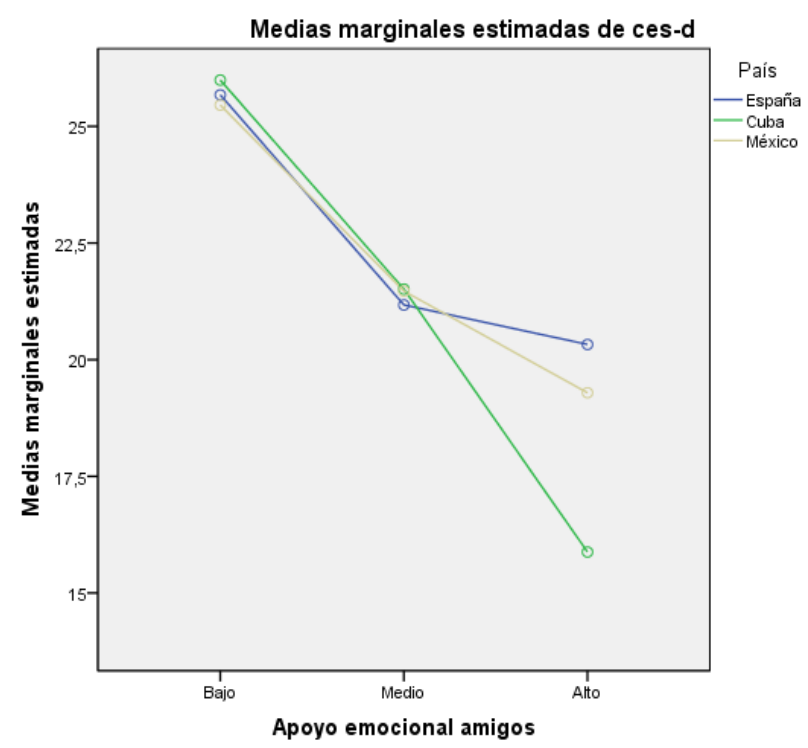

Figura 4. Interacción entre apoyo emocional de los amigos y país respecto a la depresión.

En cuanto al apoyo emocional de los amigos (AEA, en adelante), encontramos diferencias significativas en AEA con depresión, así como una clara tendencia a la significación con respecto a las diferencias relacionadas con la interacción de AEA respecto al país de pertenencia, a la par que con país y edad simultáneamente. En este sentido, mientras la relación negativa entre AEA y depresión era la esperada inicialmente, en el caso de la interacción entre AEA y país aparece una especial relevancia de la disposición de elevado AEA en la muestra cubana.

Respecto a la interacción entre AEA, país y edad, pueden apreciarse las diferencias a lo largo de los tres grupos de edad en cada país según el nivel de AEA en las figuras 5.1. a 5.3.

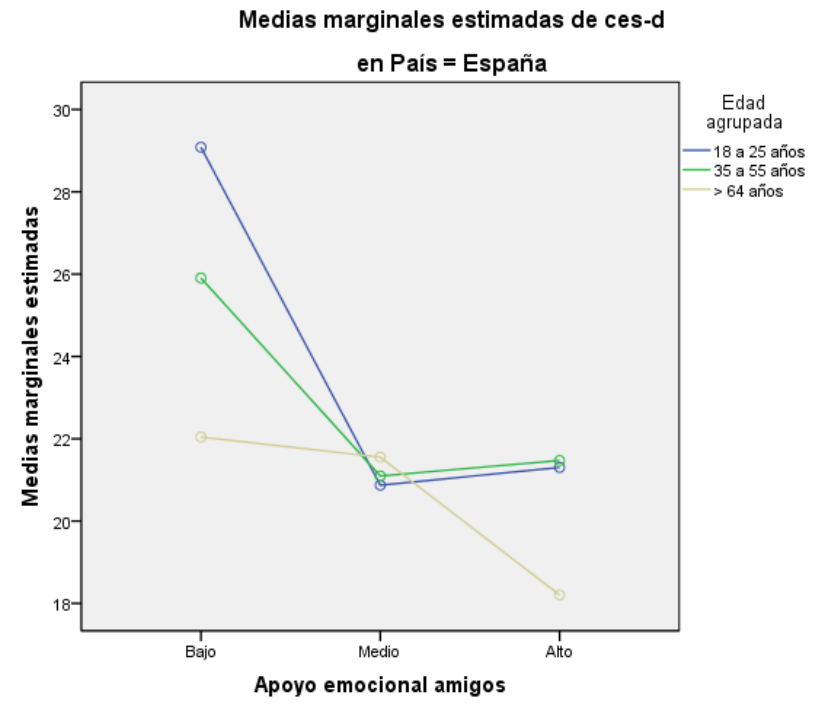

Figura 5.1. Interacción entre apoyo emocional de los amigos, edad y país (España).

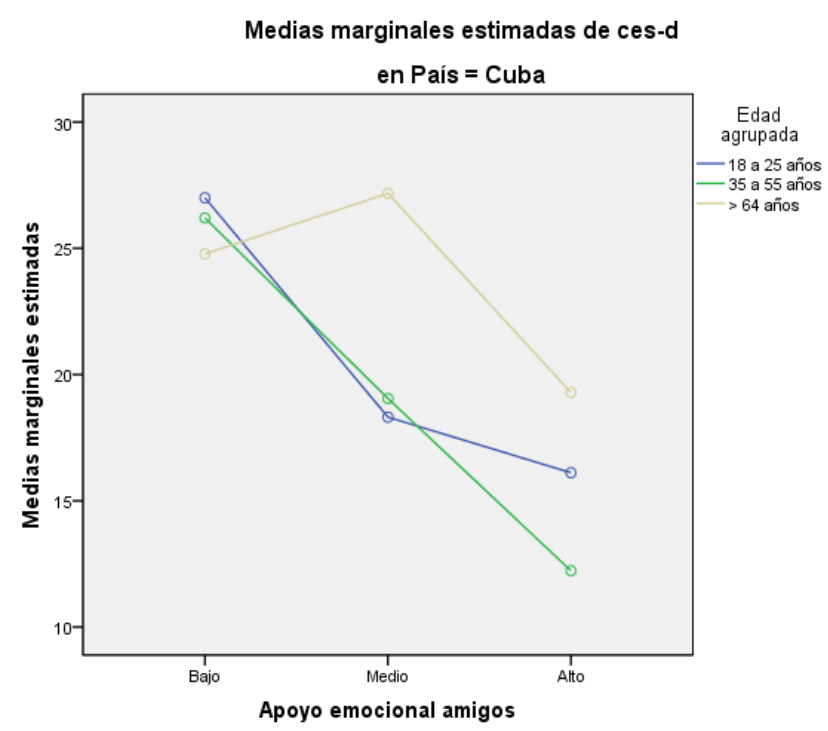

Figura 5.2. Interacción entre apoyo emocional de los amigos, edad y país (Cuba). 


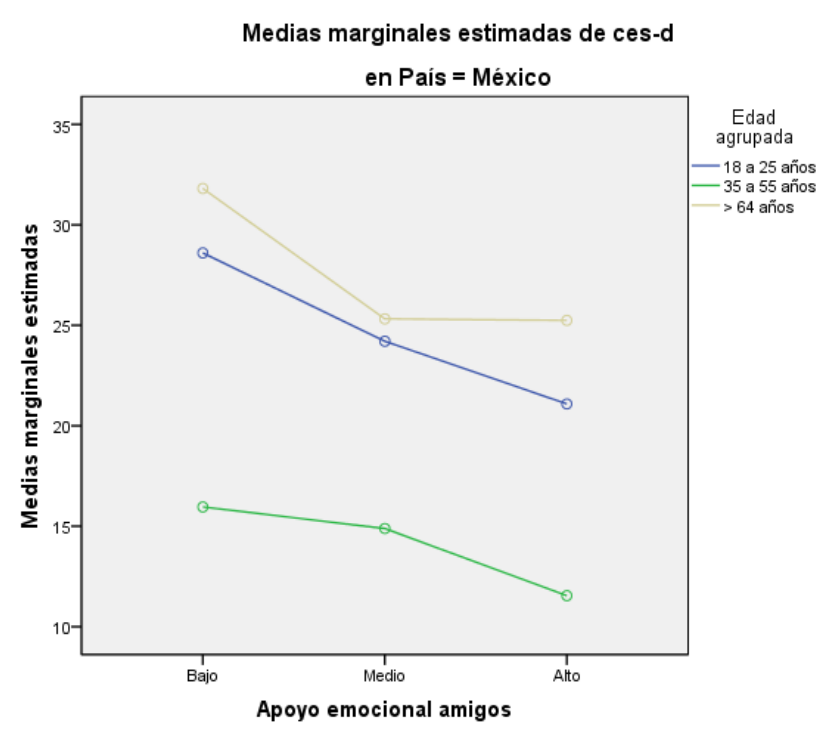

Figura 5.3. Interacción entre apoyo emocional de los amigos, edad y país (México).

El apoyo emocional de los compañeros (AEC, en adelante) muestra una relación inversa significativa en cuanto a la depresión, así como la interacción entre país y AEC respecto a depresión. En este sentido, las puntuaciones en depresión desciende de manera especialmente relevante en el caso de la muestra tomada de población cubana conforme se eleva el nivel de apoyo emocional percibido (véase Figura 6).

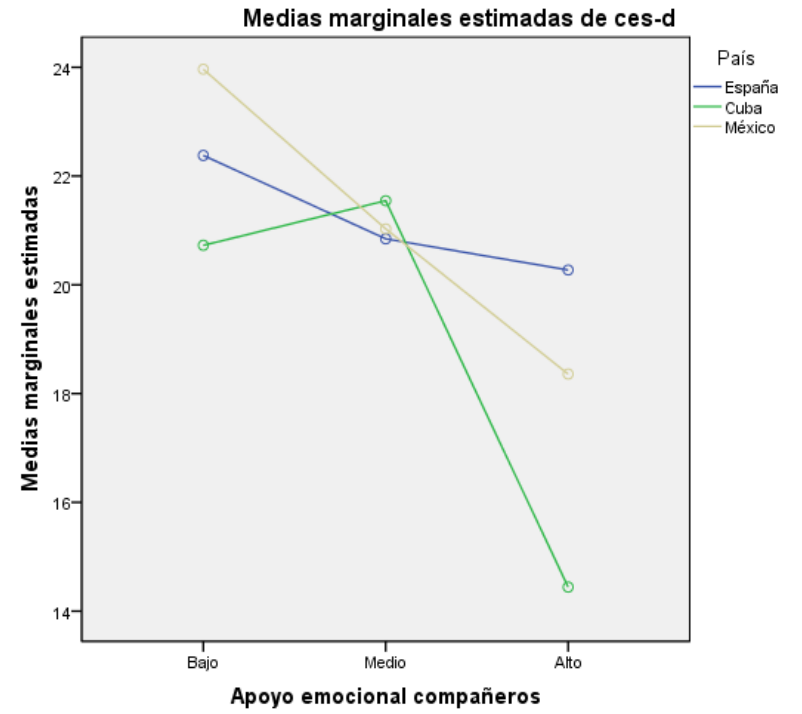

Figura 6. Interacción entre apoyo emocional de los compañeros y país respecto a la depresión.

Por lo que se refiere al apoyo emocional de los vecinos (AEV, en adelante), además de la esperada relación negativa entre esta fuente de apoyo y depresión, hemos obtenido una interacción significativa con la variable país, resaltando en este caso (véase Figura 7) cómo las puntuaciones en depresión son mas elevadas en la muestra de México entre aquellas personas que presentan un bajo nivel de AEV.

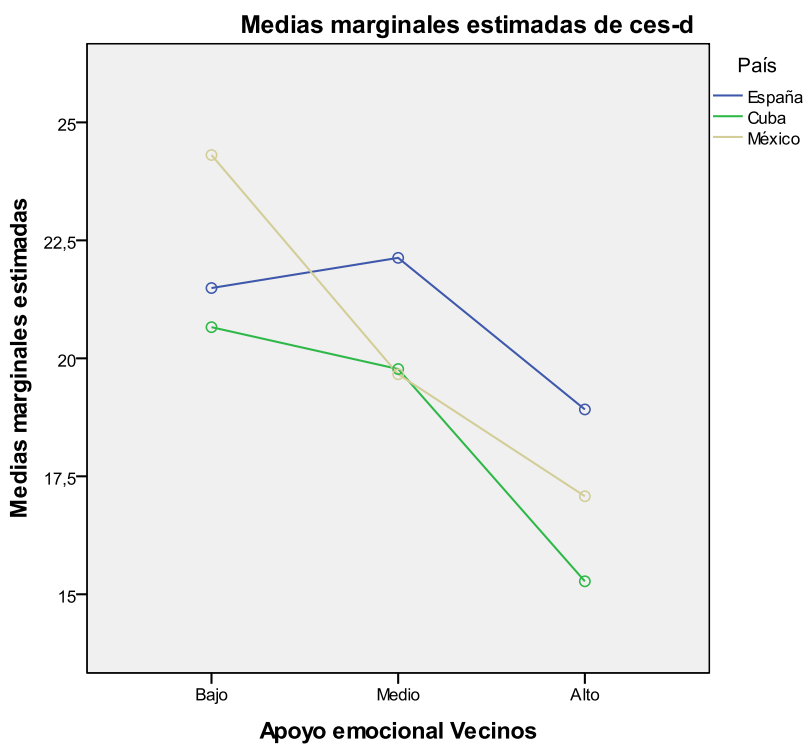

Figura 7. Interacción entre apoyo emocional de los vecinos y país respecto a la depresión

Por último, en lo concerniente a la satisfacción con el apoyo emocional (SAE, en adelante) y su relación con la depresión, además de la esperada relación negativa entre estas dos variables, encontramos una interacción significativa con el país de pertenencia y la edad. En este sentido, las figuras 8 y 9 muestran respectivamente, cómo la baja satisfacción con el apoyo emocional percibido se relaciona en mayor medida con elevadas puntuaciones en depresión en la muestra española, y la mayor relevancia de esta baja satisfacción entre las personas más jóvenes.

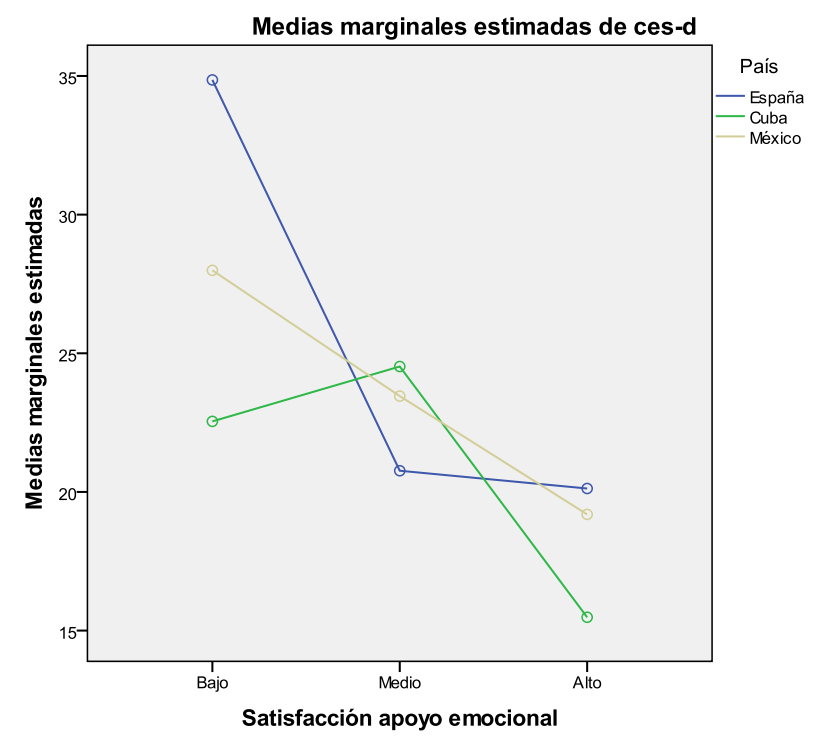

Figura 8. Interacción entre Satisfacción con el apoyo emocional percibido y país respecto a la depresión. 


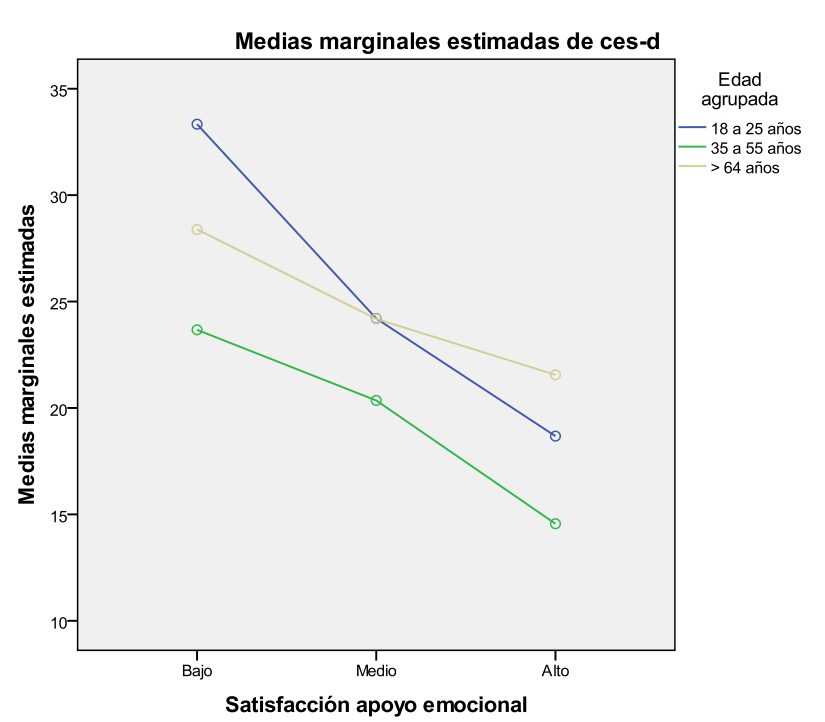

Figura 9. Interacción entre Satisfacción con el apoyo emocional percibido y edad.

\section{Discusión}

Los resultados obtenidos en el presente trabajo indican que la relación obtenida entre apoyo emocional según distintas fuentes y los valores en sintomatología depresiva varía en función de las influencias culturales asociadas al país de pertenencia de los sujetos entrevistados, a su edad, o a una interacción entre ambos elementos.

Un primer dato a destacar es que las cinco fuentes de apoyo emocional consideradas, así como la satisfacción en general con el mismo, aparecen significativamente asociadas a la sintomatología depresiva en los tres países estudiados y en los diferentes grupos de edad. No obstante, junto a esto, es necesario tener en cuenta que cada una de estas fuentes de apoyo ha mostrado una influencia relevante en su relación con alguna de las otras variables consideradas en el estudio o con su interacción.

Respecto a la variable edad, considerada en forma aislada, como ocurre con respecto a la satisfacción con el apoyo emocional percibido, o mediante su interacción con el país, como ocurre en el caso del AEP o AEA, ha mostrado que necesariamente ha de ser tenida en cuenta si queremos entender las variaciones en la eficacia del apoyo social en la prevención de problemas de salud en general, y de alteraciones depresivas, en particular. Por tanto, de acuerdo con otros trabajos previos (p. ej., Lynch, 1995) podemos indicar la relevancia de la mediación de la variable edad en la eficacia de

\section{Referencias}

Aranda, C. y Pando, M. (2013). Conceptualización del apoyo social y las redes de apoyo social. Revista de investigación en psicología, 16, (1), 233-245. Ayers, J.W., Hofstetter, R., Usita, P., Irvin, V.L., Kang, S. y Hovell, F. (2009). Sorting out the competing effects of acculturation, immigrant distintas fuentes de apoyo emocional. En este sentido, nuestros resultados son consistentes con los obtenidos por otros autores (Olsen, Iversen y Sabroe, 1991) al indicar la existencia de variaciones tanto en el nivel del apoyo social proveniente de la pareja, como en su capacidad operativa en función de la edad.

Por lo que se refiere a la influencia de los factores asociados al país de pertenencia, esta variable ha mostrado una interacción significativa con apoyo emocional respecto a la depresión, en el apoyo proveniente de la pareja, los amigos, los compañeros y los vecinos, así como con la satisfacción con el apoyo emocional. Estos resultados vienen a abundar en la necesidad de considerar la importancia de los aspectos culturales para obtener una mejor comprensión de las diferencias referidas a los procesos de estrés, afrontamiento y apoyo social, como ya ha sido indicado por otros autores (Ogawa, 2009).

Además de las implicaciones a las que hemos aludido relativas a la edad o al país de pertenencia, los datos de nuestro trabajo muestran una interacción significativa de estas variables conjuntamente consideradas con respecto al apoyo emocional y su relación con la depresión, en el caso del apoyo que proviene de la pareja y los amigos. De acuerdo con VonDras (2008), consideramos que es necesario tener en cuenta la interacción entre los factores de tipo individual y los factores de tipo cultural en el estudio relativo a la percepción, disposición y funcionalidad del apoyo social respecto a la salud.

Otro dato interesante aportado en este trabajo tiene que ver con las diferencias encontradas en depresión en relación a las muestras de los distintos países y a los grupos de edad. En este sentido, es de especial interés la interacción entre país y grupo de edad con respecto a los valores en sintomatología depresiva. Aunque este no es un objetivo específico del presente trabajo, estos datos redundan en la necesidad de referenciar el desarrollo adulto o envejecimiento en un marco coyuntural concreto para poder entender su relación con la presencia de indicadores de depresión.

Por último, quisiéramos señalar que si por lo general los datos aportados por este tipo de estudios terminan generando más preguntas que respuestas, este caso no es una excepción. No es objeto del presente estudio abordar las razones de tipo social, económico, cultural e incluso histórico que puedan fundamentar las diferencias encontradas, pero creemos que este tipo de trabajos son necesarios para poder profundizar en al análisis de las razones que llevan a la determinación de las realidades particulares de cada grupo humano.

stress, and social support on depression: A report on Korean women in California. Journal of Nervous and Mental Disease, 197(10), 742-747.

Barra, E., Cerna, R., Kramm, D. y Véliz, V. (2006). Problemas de salud, estrés, afrontmaiento, depresión y apoyo social en adolescentes. Terapia Psicológica. Revista Chilena de Psicología Clínica, 24 (1), 55-61. 
Barerra, M., Jr. (1986). Distinctions between social support concepts, measures, and models. American Journal of Community Psychology, 14, 413445.

Blackwell, E., y Conrod, P. J. (2003). A five-dimensional measure of drinking motives. Manuscrito no publicado, Department of Psychology, University of British Columbia, Vancouver, Canada.

Brannana, D., Biswas-Dienerb,R., Mohrb,C., Mortazavic, S. y Steinb, N. (2013). Friends and family: A cross-cultural investigation of social support and subjective well-being among college students. The Journal of Positive Psychology: Dedicated to furthering research and promoting good practice, $8,(1), 65-75$.

Burke, A. y Neimeyer, R.A. (2010). African American homicide bereavement: Aspects of social support that predict complicated grief, PTSD, and depression. Omega: Journal of Death and Dying, 61(1), 1-24.

Calvo, F., Díaz, M.D., Ojeda, B., Ramal, J. y Alemán, S. (2002) Diferencias en ansiedad, depresión, estrés y apoyo social entre sujetos con enfermedad cardiovascular y controles sanos. En F. Calvo (Comp.), Investigaciones en psicocardiología. Las Palmas de Gran Canaria: Colegio Oficial de Psicólogos de Las Palmas.

Cohen, S. (1988). Psychosocial models of social support in the etiology of physical disease. Health Psychology, 7, 269-297.

Campo-Arias, A., Díaz-Martínez, L., Rueda-Jaimes, G., del Pilar CadenaAfanador, L., y Hernández, N. (2007). Psychometric properties of the CES-D Scale among Colombian adults from the general population. Revista Colombiana de Psiquiatría, 36(4), 664-674.

Cassel, J. (1974). Social science in epidemiology: Psychosocial processes and stress theoretical formulation. International Journal of Helath Services, 4, 537-549.

Castellano, C.L. (2014). La influencia del apoyo social en el estado emocional y las actitudes hacia la vejez y el envejecimiento en una muestra de ancianos. International Journal of Psychology and Psychological Therapy, 2014, 14, 3, 365-377

Chen, W., Siu, O. Lu, J. Cooper, L. y Philips, R. (2009). Work stress and depression: The direct and moderating effects of informal social support and coping. Stress and Health: Journal of the International Society for the Investigation of Stress, Vol 25(5), 431-443.

Cobb, S. (1976). Social support as a moderator of life stress. Psychosomatic Medicine, 38, 300-314.

Cohen, S, y McKay, G (1984). Social support, stress, and the buffering hypothesis An empirical review In A Baum, J E Singer, y S E Taylor (Eds), Handbook of psychology and health (Vol 4, pp 253-267) Hillsdale, NJ Erlbaum

Cohen, S y Syme, S.L. (1985). Issues in the study and application of social support. In S. Cohen y S. L. Syme (Eds.), Social support and health (pp 322). San Francisco: Academic Press.

Durkheim, E. (1951). Suicide. Nueva York: Free Press.

Diaz Veiga, P. (1987). Evaluación del apoyo social. En: R. FernándezBallesteros. (Ed). El Ambiente. Análisis Psicológico. Pirámide. Madrid. 125-149.

Faris, R.E.L., y Dunham, H.W. (1939). Mental disorders in urban areas: An ecological study of schirophrenia and other psychosis. Chicago: University Press.

Glazer, S. (2006). Social support across cultures. International Journal of Intercultural Relations, 30(5), 605-622.

Goodwin, R. y Giles, S. (2003). Social support provision and cultural values in Indonesia and Britain. Journal of Cross-Cultural Psychology, 34(2), 240245

Hobfoll, S.E. y Stokes, J.P. (1988). The processes and mechanics of social support. En S. Duck (Comp.) Personal Relationships. Theory Research and Intervention. Londres: Willey and Son.

House, J. S. (1981). Work stress and social support. Reading, MA: AddisonWesley.

House, J. S., y Kahn, R. L. (1985). Measures and concepts of social support. In S. Cohen y S. L. Syme (Eds.), Social support and health (pp. 83-108). New York: Academic Press.

Khan, R.L. y Antonucci, T.C. (1980). Convoys over the life course: Attachment, roles and social support. En P. Baltes y O. Brim (Comp.), Life Span Development and Behaviour (pp. 253-286). San Diego, CA: Academic Press.
Lin, N. (1986). Conceptualizing social support. In N. Lin, A. Dean, y W. Ensel (Eds.), Social support, life events, and depression (pp. 17-30). New York: Academic Press.

Lin, N., Dean, A. y Ensel, W. M, (Eds.) (1986). Social support, life events and depression. Londres: Academic Press.

Lin, N., y Ensel, W. (1989). Life stress and health: Stressors and resources. American Sociological Review, 54, 382-399.

Lynch, S. (1995). Age and gender differences in the structure, function, and perceived quality of social support convoys. Humanities and Social Sciences, 55(8-A).

Martín, M., Riquelme, A., Pérez, R., Enríquez, J.A. y Ortigosa, J. (2010). Revista del Hospital Psiquiátrico de la Habana, 7 (1).

Martínez, M.F., García, M. y Maya, I. (2001). El efecto amortiguador del apoyo social sobre la depresión en un colectivo de inmigrantes. Psicothema, 13 (4), 605-610.

Norbeck, J. S., Lindsey, A. M. y Carrieri, V. L (1983). The development of an instrument to measure social support. Nursing Research, 30 (3), 264-

Nolen-Hoesksema, S. y Aldao, A. (2011). Gender and age differences in emotion regulation strategies and their relationship to depressive symptoms. Personality and individual differences, 51, (6), 704-708.

Ogawa, N. (2009). Stress, coping behavior, and social support in Japan and the United States. Social Processes y Social Issues, 69.

O'Leary, E., Murphy, M. y Chen, C. (2009). A cross-cultural investigation of social support in adults over 60 in Ireland and China. Journal of Psychology in Chinese Societies, 10 (1), 39-55

Olsen, O., Iversen, L. y Sabroe, S. (1991). Age and the operationalization of social support. Social Science y Medicine, 32 (7), 767-771.

Piko, B.F., Luszczynska, A. y Fitzpatrick, K.M. (2012). Social inequalities in adolescent depression: The role of parental social support and optimism. International Journal of Social Psychiatry, 59 (5) 474-481.

Radloff, L. (1977). The CES-D Scale: A Self-Report Depression Scale for Research in the General Population. Applied Psychological Measurement, 1(3), 385-401.

Riquelme, A., Martín, M., Carrillo, M.J., y López, A. I. (2010). Apoyo social en ancianos. En Riquelme, A., Ortigosa, J.M. y Martín, M. (Eds.) Manual de psicogerontología. Madrid. Ediciones Académicas.

Riquelme, A. (2012). Utilidad y características psicométricas de un modelo de autoinforme para la evaluación del apoyo social percibido. Manuscrito no publicado. Departamento de Personalidad, Evaluación y Tratamiento Psicológi cos. Universidad de Murcia, España.

Robles, R., Morales, M., Jiménez, L. y Morales, J. (2009). Depresión y ansiedad en mujeres con cáncer de mama: El papel de la afectividad y el soporte social. Psicooncología, 6 (1), 191-201.

Sahar, G. Gohen, G., Grogan, E., Braile, J.P. y Henrich, Ch. (2009). Terrorism-related perceived stress, adolescent depression, and social support from friends. Pediatrics, 124 (2), 235-240.

Samter, W., Whaley, B. B., Mortenson, S. T. y Burleson, B. R. (1997). Ethnicity and emotional support in same-sex friendship: A comparison of Asian-Americans, African-Americans, and Euro-Americans. Personal Relationships, 4(4), 413-430.

Schumm, J.A., Briggs, M. y Hobfoll, E. (2006). Cumulative interpersonal traumas and social support as risk and resiliency factors in predicting PTSD and depression among inner-city women. Journal of Traumatic Stress, 19(6), 825-836.

Sherbourne, C.D. y Stewart, A.L. (1991). The MOS Social Support Survey. Social Science y Medicine, 32, 705-714.

Shery, S., Law, A., Hewitt, P.L., Flett, L. y Besser, A. (2008). Social support as a mediator of the relationship between perfectionism and depression: A preliminary test of the social disconnection model. Personality and Individual Differences, 45 (5), 339-344.

Thomas, W.I., y Florian Znaniecki (1920). The Polish Peasant in Europe and America. Boston: Badger Press.

Vargas, A. y Arnett, P. (2010). Positive everyday experiences interact with social support to predict depression in multiple sclerosis. Journal of the International Neuropsychological Society, 16(6), 1039-1046.

VonDras, D., Pouliot, G., Malcore, S. y Iwahashi, S. (2008). Effects of Culture and Age on the Perceived Exchange of Social Support Resources. The International Journal of Aging and Human Development, 67(1), 63-100. 
Vyavaharkar, M., Moneyham,L, Corwin, S., Saunders, R., Annang, L. y Tavakoli, A. (2010). Relationships between stigma, social support, and depression in HIV-infected African American women living in the rural southeastern United States. Journal of the Association of Nurses in AIDS Care, 21 (2), 144-152.
Zunzunegui, V., Delgado, M., Pérez, E., Yague, A., Illescas, M. y León, V. (1998). Validación de la escala Ces-d para la medida de la sintomatología depresiva en una población de personas mayores españolas. Revista multidisciplinar de gerontologia, 8 (3), 151-161.

(Artículo recibido: 28-07-2014; revisado: 19-11-2014; aceptado: 06-02-2015) 
Anexo. Cuestionarios sobre apoyo emocional percibido

\section{(A. Riquelme, 2012)}

Departamento de Personalidad, Evaluación y Tratamientos Psicológicos. Universidad de Murcia

Diga en qué medida estas personas le hacen sentirse querido, amado, valorado, respetado, etc.

0 Nada/ 1 Un poco/ 2Moderadamente/ 3 Mucho/ 4 totalmente

\begin{tabular}{|c|c|c|}
\hline Personas & Nivel de apoyo & No procede \\
\hline Pareja & $\begin{array}{lllll}0 & 1 & 2 & 3 & 4\end{array}$ & \\
\hline Familares & $\begin{array}{lllll}0 & 1 & 2 & 3 & 4\end{array}$ & \\
\hline Amigos & $\begin{array}{lllll}0 & 1 & 2 & 3 & 4\end{array}$ & \\
\hline Compañeros & $\begin{array}{lllll}0 & 1 & 2 & 3 & 4\end{array}$ & \\
\hline Vecinocs & $\begin{array}{lllll}0 & 1 & 2 & 3 & 4\end{array}$ & \\
\hline Otras, ¿cuáles? & $\begin{array}{lllll}0 & 1 & 2 & 3 & 4\end{array}$ & \\
\hline Su nivel de satisfacción en general con el apoyo emocional del que dispone es: & $\begin{array}{lllll}0 & 1 & 2 & 3 & 4\end{array}$ & \\
\hline
\end{tabular}

